PROCEEDINGS OF THE

AMERICAN MATHEMATICAL SOCIETY

Volume 125, Number 10, October 1997, Pages 2825-2830

S 0002-9939(97)03987-7

\title{
ON THE INTERSECTION PROPERTY OF DUBROVIN VALUATION RINGS
}

\author{
ZHAO YICAI
}

(Communicated by Ken Goodearl)

\begin{abstract}
It is shown that of the three axioms Gräter specified for his intersection property of Dubrovin valuation rings in central-simple algebras, the second and third axioms actually follow from the first.
\end{abstract}

\section{INTRODUCTION}

In [G1] Gräter introduced the intersection property of Dubrovin valuation rings which proved to be a crucial property in studying extensions of valuation rings and the structure of intersections of a finite number of Dubrovin valuation rings. In that paper a numerical invariant was obtained which has a close relation to the intersection property and this invariant was equal to that of the Ostrowski Theorem for Dubrovin valuation rings in [W], and so a new Defektsatz was stated. On the other hand, the intersection property is equivalent to Morandi's condition which is necessary and sufficient to get the general approximation theorem in [M1]. Furthermore, any semilocal Bézout order in a central simple algebra is precisely an intersection of a finite number of Dubrovin valuation rings having the intersection property [G2]. Thus Dubrovin valuation rings with the intersection property form a useful tool in studying prime PI-Bézout rings.

The purpose of this paper is to show that the intersection property can be replaced by a simpler property, i.e., property P. It turns out to be convenient in studying Dubrovin valuation rings by using property $\mathrm{P}$.

This paper makes strong use of the results of [G1] and [M3].

\section{Preliminaries}

Throughout this paper $Q$ will denote a central simple algebra with finite dimension over its center $F$. If $R$ is any ring, then $Z(R)$ is the center of $R, J(R)$ the Jacobson radical of $R, R^{*}$ the group of units of $R$ and $\operatorname{Spec}(R)$ the set of all prime ideals of $R$. A subring $B$ of $Q$ is called a Dubrovin valuation ring of $Q$ if $B$ has an ideal $I$ such that $B / I$ is simple Artinian and for any $q \in Q \backslash B$ there exist $b, b^{\prime} \in B$ with $b q, q b^{\prime} \in B \backslash I$. It is shown in [D1] that $I=J(B)$, the only maximal ideal of $B$, and that $Z(B)=B \cap F$, a valuation ring of $F$. Other properties of Dubrovin valuation rings can be found in [D1], [D2], [W] and [G1]. Let $B$ be a Dubrovin

Received by the editors December 18, 1995 and, in revised form, March 29, 1996.

1991 Mathematics Subject Classification. Primary 16A40, 16A10.

(C) 1997 American Mathematical Society 
valuation ring of $Q$, then $\mathcal{B}(B)$ denotes the set of all overrings of $B$ in $Q$. In this paper, $\subseteq$ stands for inclusion and $\subset$ for proper inclusion.

Following Gräter [G1] the intersection property is defined as follows.

Definition. Let $B_{1}, \ldots, B_{n}$ be Dubrovin valuation rings of $Q$ and let $R=B_{1} \cap$ $\cdots \cap B_{n}$. Then $B_{1}, \ldots, B_{n}$ have the intersection property if

$$
\begin{aligned}
f: \mathcal{B}\left(B_{1}\right) \cup \cdots \cup \mathcal{B}\left(B_{n}\right) & \rightarrow \operatorname{Spec}(R) \\
B & \mapsto J(B) \cap R
\end{aligned}
$$

is a well-defined anti-order-isomorphism.

In other words, we say that $B_{1}, \ldots, B_{n}$ have the intersection property if the following conditions are satisfied:

(1) For any overring $B$ of $B_{i}$ in $Q(i=1, \ldots, n), J(B) \cap R$ is a prime ideal of $R$.

(2) For any prime ideal $P$ of $R$, there exists a unique $B \in \mathcal{B}\left(B_{i}\right)$ for some $i=1, \ldots, n$ such that $P=J(B) \cap R$.

(3) For any $B, B^{\prime} \in \mathcal{B}\left(B_{1}\right) \cup \cdots \cup \mathcal{B}\left(B_{n}\right), B \subseteq B^{\prime}$ if and only if $J\left(B^{\prime}\right) \cap R \subseteq$ $J(B) \cap R$.

Definition. Let $B_{1}, \ldots, B_{n}$ be Dubrovin valuation rings of $Q$ and let $R=B_{1} \cap \cdots \cap$ $B_{n}$. Then $B_{1}, \ldots, B_{n}$ have property $\mathrm{P}$ if for any overring $B$ of $B_{i}$ in $Q(i=1, \ldots, n)$, $J(B) \cap R$ is a prime ideal of $R$.

We will show that the intersection property is equivalent to property $\mathrm{P}$. This means that conditions (2) and (3) follow from condition (1).

\section{Proof of the MAIN THEOREM}

First of all, we need some lemmas.

Lemma 1. Let $B_{1}, \ldots, B_{n}$ be Dubrovin valuation rings of $Q$ and let $R=B_{1} \cap \cdots \cap$ $B_{n}$ and $D=R \cap F$.

(i) Let $S \neq \varnothing$ be a multiplicative subset of $D$ and $I$ an ideal of $R_{s}$. Then $I$ is a prime ideal of $R_{s}$ if and only if $I \cap R$ is a prime ideal of $R$, where $R_{s}=R S^{-1}$, the localization of $R$ at $S$.

In particular, if $B_{1}, \ldots, B_{n}$ have property $\mathrm{P}$, then $B_{1 s}, \ldots, B_{n s}$ have property $\mathrm{P}$.

(ii) $B_{1}, \ldots, B_{n}$ have property $\mathrm{P}$ if and only if $B_{1 m}, \ldots, B_{n m}$ have property $\mathrm{P}$ for all maximal ideals $m$ of $D$, where $B_{i m}=B_{i}(D \backslash m)^{-1}$.

Proof. (i) Suppose $I$ is a prime ideal of $R_{s}$. For any $a, b \in R$, if $a R b \subseteq I \cap R$, then $a R_{s} b \subseteq(I \cap R)_{s}=I$. Hence $a$ or $b \in I$ and then $a$ or $b \in I \cap R$. It follows that $I \cap R$ is a prime ideal of $R$.

Conversely, suppose $I \cap R$ is a prime ideal of $R$. For any $x, y \in R_{s}$, we have $x=u s^{-1}$ and $y=v t^{-1}$ for some $u, v \in R$ and some $s, t \in S$. If $x R_{s} y \subseteq I$, then $u R_{s} v \subseteq I$. So $u R v \subseteq I \cap R$. Since $I \cap R$ is a prime ideal of $R, u$ or $v \in I \cap R$. Thus $x$ or $y \in(I \cap R)_{s}=I$. Therefore $I$ is a prime ideal of $R_{s}$.

Finally, assume that $B_{1}, \ldots, B_{n}$ have property P. For any given $B_{i s}$, if $B \in$ $\mathcal{B}\left(B_{i s}\right)$ then $B \in \mathcal{B}\left(B_{i}\right)$. By assumption, $J(B) \cap R$ is a prime ideal of $R$. It follows that $J(B) \cap R_{s}$ is a prime ideal of $R_{s}$, as $\left(J(B) \cap R_{s}\right) \cap R=J(B) \cap R$. Hence $B_{1 s}, \ldots, B_{n s}$ have property P.

(ii) Suppose $B_{1 m}, \ldots, B_{n m}$ have property $\mathrm{P}$ for all maximal ideals $m$ of $D$. For any $B_{i}$, write $V_{i}=Z\left(B_{i}\right)$; then $J\left(V_{i}\right) \cap D$ is a prime ideal of $D$. There exists a maximal ideal $m$ of $D$ containing $J\left(V_{i}\right) \cap D$. We have $B_{i m}=B_{i}(D \backslash m)^{-1} \subseteq$ 
$B_{i}\left(D \backslash J\left(V_{i}\right)\right)^{-1} \subseteq B_{i}\left(V_{i} \backslash J\left(V_{i}\right)\right)^{-1}=B_{i}$. If $B \in \mathcal{B}\left(B_{i}\right)$, then $B \in \mathcal{B}\left(B_{i m}\right)$. By assumption, $B_{1 m}, \ldots, B_{n m}$ have property P. Hence $J(B) \cap R_{m}$ is a prime ideal of $R_{m}$. By (i) $J(B) \cap R$ is a prime ideal of $R$ as $\left(J(B) \cap R_{m}\right) \cap R=J(B) \cap R$. Therefore $B_{1}, \ldots, B_{n}$ have property $\mathrm{P}$.

The converse follows from (i).

Lemma 2. Let $B_{1}, \ldots, B_{n}, B$ be Dubrovin valuation rings of $Q$ such that $B_{1}, \ldots, B_{n}$ $\subseteq B$. Then $B_{1}, \ldots, B_{n}$ have property $\mathrm{P}$ if and only if $B_{1} / J(B), \ldots, B_{n} / J(B)$ have property $\mathrm{P}$.

Proof. Let $R=B_{1} \cap \cdots \cap B_{n}$. Assume that $B_{1}, \ldots, B_{n}$ have property P. $B_{1} / J(B)$, $\ldots, B_{n} / J(B)$ are Dubrovin valuation rings of $B / J(B)$. For any given $B_{i}$, if $C^{\prime} \in$ $\mathcal{B}\left(B_{i} / J(B)\right)$, then there exists a Dubrovin valuation $\operatorname{ring} C$ of $Q$ with $B_{i} \subseteq C \subseteq B$ such that $C^{\prime}=C / J(B)$. By assumption, $J(C) \cap R$ is a prime ideal of $R$. Then $J(C) / J(B) \cap R / J(B)$ is a prime ideal of $R / J(B)$. Hence $B_{1} / J(B), \ldots, B_{n} / J(B)$ have property $\mathrm{P}$.

Conversely, assume that $B_{1} / J(B), \ldots, B_{n} / J(B)$ have property P. For any $C \in$ $\mathcal{B}\left(B_{i}\right)$, if $C \subseteq B$, then $C / J(B) \in \mathcal{B}\left(B_{i} / J(B)\right)$. By assumption, $J(C) / J(B) \cap$ $R / J(B)$ is a prime ideal of $R / J(B)$. Hence $J(C) \cap R$ is a prime ideal of $R$. If $C \nsubseteq B$, then $B \subset C$ as $\mathcal{B}\left(B_{i}\right)$ is totally ordered by inclusion. Now $B_{1} / J(C), \ldots, B_{n} / J(C)$ are Dubrovin valuation rings of $C / J(C)$. By [K, Theorem 107], $Z\left(B_{i} / J(C)\right)$ has

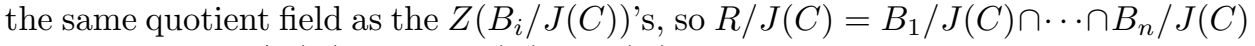
is an order in $C / J(C)$, hence $J(C)=J(C) \cap R$ is a prime ideal of $R$. Therefore $B_{1}, \ldots, B_{n}$ have property $\mathrm{P}$.

Lemma 3. Let $B_{1}, \ldots, B_{n}$ be Dubrovin valuation rings of $Q$ having property $\mathrm{P}$ such that $V=B_{1} \cap F=\cdots=B_{n} \cap F$. If each $B_{i}$ is integral over $V$, then $B_{1}=\cdots=B_{n}$.

Proof. Suppose $B_{i} \neq B_{j}$ for some $B_{i}$ and $B_{j}$. We can assume that $B_{1}, \ldots, B_{n}$ are incomparable. For any $B_{i}, i=1, \ldots, n, B_{1}$ and $B_{i}$ are conjugate by $[\mathrm{W}$, Theorem A], that is, there exist $q_{i} \in Q^{*}$ such that $B_{i}=q_{i} B_{1} q_{i}^{-1}$ for $i=1, \ldots, n$. We first note that for any $q \in Q^{*}$, there exists $v \in \operatorname{st}\left(B_{1}\right)=\left\{x \in Q^{*}, x B_{1}=B_{1} x\right\}$ such that $q v \in B_{1} \backslash J\left(B_{1}\right)$. In fact, since $B_{1}$ is integral over $V$, by [W, Theorem $\mathrm{F}$ ] there exists $a \in \operatorname{st}\left(B_{1}\right)$ such that $B_{1} q B_{1}=a B_{1}$, i.e., $B_{1} q a^{-1} B_{1}=B_{1}$. Let $v=a^{-1}$; then $q v \in B_{1} \backslash J\left(B_{1}\right)$ and $v \in \operatorname{st}\left(B_{1}\right)$. Now, for each $q_{i}$, there exists a $v_{i} \in \operatorname{st}\left(B_{1}\right)$ such that $q_{i} v_{i} \in B_{1} \backslash J\left(B_{1}\right)$. By replacing $q_{i} v_{i}$ by $q_{i}$, we may assume $q_{i} \in B_{1} \backslash J\left(B_{1}\right)$. For $q_{i}^{-1}$, there exists a $u_{i} \in \operatorname{st}\left(B_{1}\right)$ such that $q_{i}^{-1} u_{i} \in B_{1} \backslash J\left(B_{1}\right)$. Hence $q_{i}^{-1} u_{i} B_{1} q_{i} \subseteq B_{1}$ and then we have that $u_{i} B_{1} \subseteq q_{i} B_{1} q_{i}^{-1}=B_{i}$ for $i=1, \ldots, n$. Set $I_{1}=\bigcap u_{i} B_{1}$ and $R=B_{1} \cap \cdots \cap B_{n}$; then $I_{1}$ is an ideal of $R$. Since $q_{i}^{-1} u_{i}, q_{i} \in B_{1} \backslash J\left(B_{1}\right), u_{i} B_{1}=B_{1} u_{i}$ is a two-sided ideal of $B_{1}$. By [D1, Theorem 4] the $u_{i} B_{1}$ are totally ordered by inclusion. Thus there exists some $u_{i_{1}}$ in $\left\{u_{1}, \ldots, u_{n}\right\}$ such that $I_{1}=u_{i_{1}} B_{1}$ and $B_{i_{1}}=q_{i_{1}} B_{1} q_{i_{1}}^{-1}$. Similarly, we have that for each $B_{k} \in\left\{B_{1}, \ldots, B_{n}\right\}$ there exists a $u_{i_{k}} \in \operatorname{st}\left(B_{k}\right), q_{i_{k}} \in Q^{*}$ and $B_{i_{k}} \in\left\{B_{1}, \ldots, B_{n}\right\}$ such that $B_{i_{k}}=q_{i_{k}} B_{k} q_{i_{k}}^{-1}$ and $u_{i_{k}} B_{k}$ is a two-sided ideal of $R=B_{1} \cap \cdots \cap B_{n}$ and $q_{i_{k}}, q_{i_{k}}^{-1} u_{i_{k}} \in B_{k} \backslash J\left(B_{k}\right)$. Set $P_{i}=J\left(B_{i}\right) \cap R$ for $i=1, \ldots, n$. Then each $P_{i}$ is a prime ideal of $R$ by assumption. Suppose that for each $B_{k} \in\left\{B_{1}, \ldots, B_{n}\right\}, u_{i_{k}} B_{k} \nsubseteq P_{i_{k}}$. Because $B_{k}$ and $R$ have a common ideal $u_{i_{k}} B_{k}$ which does not lie in $P_{i_{k}}$, there is by [AS, Theorem 2.5] a prime ideal $P_{k}^{\prime}$ of $B_{k}$ such that $P_{i_{k}}=P_{k}^{\prime} \cap R$. Thus $P_{i_{k}} \subseteq J\left(B_{k}\right) \cap$ $R=P_{k}$. It follows that $P_{i_{k}} \subset P_{k}$ as $u_{i_{k}} B_{k} \subseteq J\left(B_{k}\right) \cap R=P_{k}$. Noting that 
$\left\{P_{i_{1}}, \ldots, P_{i_{n}}\right\} \subseteq\left\{P_{1}, \ldots, P_{n}\right\}$, we conclude that each $P_{k} \in\left\{P_{1}, \ldots, P_{n}\right\}$ properly contains some one of $\left\{P_{1}, \ldots, P_{n}\right\}$. This is impossible. Indeed, since $\left\{P_{1}, \ldots, P_{n}\right\}$ is finite, we may choose $P_{k}$ minimal in $\left\{P_{1}, \ldots, P_{n}\right\}$. But $P_{k}$ strictly contains $P_{i_{k}}$, a contradiction. Hence there is $B_{k}$ in $\left\{B_{1}, \ldots, B_{n}\right\}$ such that $u_{i_{k}} B_{k} \subseteq P_{i_{k}}$. Then $q_{i_{k}}^{-1} u_{i_{k}} B_{k} q_{i_{k}} \subseteq q_{i_{k}}^{-1} P_{i_{k}} q_{i_{k}} \subseteq q_{i_{k}}^{-1} J\left(B_{i_{k}}\right) q_{i_{k}}=J\left(B_{k}\right)$. Since $q_{i_{k}}^{-1} u_{i_{k}}, q_{i_{k}} \in B_{k} \backslash J\left(B_{k}\right)$, it follows that $B_{k}=B_{k} q_{i_{k}}^{-1} u_{i_{k}} B_{k} q_{i_{k}} B_{k} \subseteq B_{k} J\left(B_{k}\right) B_{k}=J\left(B_{k}\right)$, a contradiction. Therefore, $B_{1}=\cdots=B_{n}$.

By [G1, Corollary 5.3], we know that each proper Dubrovin valuation ring is contained in a proper valuation ring which is integral over its center.

Lemma 4. Let $B_{1}, \ldots, B_{n}$ be incomparable Dubrovin valuation rings of $Q$ having property $\mathrm{P}$ such that $D=F \cap B_{1} \cap \cdots \cap B_{n}$ is a valuation ring of $F$. Let $R_{i}$ be the minimal Dubrovin valuation ring of $Q$ containing $B_{i}$ such that $R_{i}$ is integral over its center $D_{i}=R_{i} \cap F$. Then $R_{1}=\cdots=R_{n}$.

Proof. Since $D$ is a valuation ring of $F$, overrings of $D$ in $F$ are totally ordered by inclusion. Now $D \subseteq D_{i}$ for $i=1, \ldots, n$, so $D_{1}, \ldots, D_{n}$ are totally ordered, say, $D_{1} \subseteq \cdots \subseteq D_{n}$. Suppose that $D_{1} \neq D_{n}$. Write $V_{i}=F \cap B_{i}$. Then either $D_{1} \subseteq V_{n}$ or $V_{n} \subset D_{1}$ as $V_{n}$ and $D_{1}$ contain $D$. If $V_{n} \subset D_{1}$, then $Z\left(D_{1} B_{n}\right)=Z\left(R_{1}\right)=D_{1}$. So $D_{1} B_{n}$ and $R_{1}$ are conjugate and then $D_{1} B_{n}$ is integral over $D_{1}$. However, $Z\left(D_{1} B_{n}\right)=D_{1} \subset D_{n}=Z\left(R_{n}\right)$. It follows that $B_{n} \subset D_{1} B_{n} \subset D_{n} B_{n}=R_{n}$. This is contrary to the minimality of $R_{n}$. Thus we have $D_{1} \subseteq V_{n}$. Since $R_{1}$ is integral over $D_{1}, R_{1} V_{n}$ is integral over $V_{n}$. Since $Z\left(R_{1} V_{n}\right)=Z\left(B_{n}\right)=V_{n}, B_{n}$ and $R_{1} V_{n}$ are conjugate by [W, Theorem A]. Hence $B_{n}$ is integral over $V_{n}$. It follows that $B_{n}=R_{n}$. Let $P=J\left(D_{n}\right)$; then $D_{n}=D_{p}$ and $\left(B_{1}\right)_{p} \cap F=\cdots=\left(B_{n}\right)_{p} \cap F=D_{n}$ as $D \subseteq B_{i} \cap F \subseteq D_{n}$. Since $B_{n_{p}}=B_{n}$ is integral over $D_{n}$, each $B_{i_{p}}$ is integral over $D_{n}$ by [W, Theorem A]. Furthermore, by Lemma 1 (i) $B_{1_{p}}, \ldots, B_{n_{p}}$ have property P. Then by Lemma $3, B_{1_{p}}=\cdots=B_{n_{p}}=B_{n}$. It follows that each $B_{i} \subseteq B_{n}$. This is a contradiction. Hence $D_{1}=\cdots=D_{n}$ and then $R_{i}=B_{i_{p}}$ for all $i$. By Lemma (i) $R_{1}, \ldots, R_{n}$ have property $\mathrm{P}$ and by Lemma $3, R_{1}=\cdots=R_{n}$.

Lemma 5. Let $B_{1}, \ldots, B_{n}, B$ be Dubrovin valuation rings of $Q$ such that $B_{i} \subseteq B$ for some $i$. Then $B_{1}, \ldots, B_{n}, B$ have property $\mathrm{P}$ if and only if $B_{1}, \ldots, B_{n}$ have property $\mathrm{P}$.

Proof. It is trivial as $B_{1} \cap \cdots \cap B_{n} \cap B=B_{1} \cap \cdots \cap B_{n}$ and $\mathcal{B}(B) \subseteq \mathcal{B}\left(B_{i}\right)$.

Lemma 6. Let $B_{1}, \ldots, B_{n}$ be Dubrovin valuation rings of $Q$ having property $\mathrm{P}$. If $B, B^{\prime}$ are Dubrovin valuation rings of $Q$ such that $B_{i} \subseteq B, B_{j} \subseteq B^{\prime}$ for some $i, j=1, \ldots, n$, then $B, B^{\prime}$ have property $\mathrm{P}$.

Proof. Set $D=F \cap B_{1} \cap \cdots \cap B_{n}$ and $W=F \cap B \cap B^{\prime}$. For any maximal ideal $m$ of $W$, by Lemma 1 (ii), $B_{1_{p}}, \ldots, B_{n_{p}}$ have property $\mathrm{P}$, where $P=m \cap D$. By [E, 11.4, 11.12], $D_{p}=F \cap B_{1_{p}} \cap \cdots \cap B_{n_{p}}$ is a valuation ring of $F$ and $B_{i_{p}} \subseteq B_{m}$, $B_{j_{p}} \subseteq B_{m}^{\prime}$. By Lemma 1(ii) again, we may assume that $D=F \cap B_{1} \cap \cdots \cap B_{n}$ is a valuation ring of $F$, to prove that $B, B^{\prime}$ have property $\mathrm{P}$. By Lemma 5 , it is enough to consider the situation that $n \neq 1$ and $B_{1}, \ldots, B_{n}$ are incomparable and $B, B^{\prime}$ are incomparable. We prove the lemma by induction on $[Q: F]$. Let $[Q: F]>1$. Let $C$ be the minimal Dubrovin valuation ring of $Q$ containing $B_{1}$ such that $C$ is integral over its center $V=F \cap C$. Then by Lemma $4, B_{1}, \ldots, B_{n} \subset C$ and by [G1, Corollary 5.6] $Z(C / J(C)) \neq V / J(V)$. Thus $[C / J(C): Z(C / J(D))]<[Q: F]$. By 
Lemma $2, B_{1} / J(C), \ldots, B_{n} / J(C)$ are Dubrovin valuation rings of $C / J(C)$ having property P. If $B$ or $B^{\prime} \supseteq C$, then $B \subseteq B^{\prime}$ or $B^{\prime} \subset B$ as $\mathcal{B}\left(B_{i}\right)$ and $\mathcal{B}\left(B_{j}\right)$ are totally ordered by inclusion. So we may assume that $B, B^{\prime} \subset C$. Now, by induction, $B / J(C), B^{\prime} / J(C)$ have property $\mathrm{P}$. Hence $B, B^{\prime}$ have property $\mathrm{P}$ by Lemma 2

Lemma 7. Let $B_{1}, B_{2}$ be Dubrovin valuation rings of $Q$ having property $P$. Then $B_{1}$ and $B_{2}$ are comaximal in $Q$ if and only if $Z\left(B_{1}\right)$ and $Z\left(B_{2}\right)$ are comaximal in $F$.

Proof. Assume that $B_{1}$ and $B_{2}$ are comaximal in $Q$. If $V \neq F$ is an overring of $Z\left(B_{1}\right)$ and $Z\left(B_{2}\right)$ in $F$, then

$$
D=Z\left(B_{1}\right) \cap Z\left(B_{2}\right)=Z\left(B_{1}\right) \cap Z\left(B_{2}\right) \cap V .
$$

By [K, Theorem 107] $V=D_{p}$ for some prime ideal $P$ of $D$. By Lemma 1(i) $B_{1_{p}}, B_{2_{p}}$ have property $\mathrm{P}$ and $Z\left(B_{1_{p}}\right)=Z\left(B_{2_{p}}\right)=V$. By [G1, Corollary 5.6] and Lemma 4, there is a Dubrovin valuation ring $B \neq Q$ containing $B_{1}$ and $B_{2}$, a contradiction. Hence $Z\left(B_{1}\right)$ and $Z\left(B_{n}\right)$ are comaximal in $F$. The converse is trivial.

Now, we can give the main theorem of this paper.

Theorem. Let $B_{1}, \ldots, B_{n}$ be Dubrovin valuation rings of $Q$. If $B_{1}, \ldots, B_{n}$ have property $\mathrm{P}$, then $B_{1}, \ldots, B_{n}$ have the intersection property.

Proof. Assume that $B_{1}, \ldots, B_{n}$ have property P. Then by Lemma 6 any $B_{i}, B_{j}$ have property $\mathrm{P}$ for $i, j=1, \ldots, n$, and by Lemma $2, B_{i} / J\left(B_{i j}\right), B_{j} / J\left(B_{i j}\right)$ have property $\mathrm{P}$, where $B_{i j}$ are the least overring of $B_{i}$ and $B_{j}$ in $Q$. By Lemma 7, $Z\left(B_{i} / J\left(B_{i j}\right)\right)$ and $Z\left(B_{j} / J\left(B_{i j}\right)\right)$ are comaximal and then by [G1, Corollary 6.2] $B_{i} / J\left(B_{i j}\right), B_{j} / J\left(B_{i j}\right)$ have the intersection property. Hence any $B_{i}, B_{j}$ have the intersection property by [G1, Prop. 6.3] and then by [G1, Theorem 6.8], $B_{1}, \ldots, B_{n}$ have the intersection property.

\section{REFERENCES}

[AD] J. H. Alajbegovic and N. I. Dubrovin, Noncommutative Prüfer rings, J. Algebra 135 (1990), 165-176. MR 92b:16019

[AS] S. A. Amitsur and L. W. Small, Prime ideals in PI-rings, J. Algebra 62 (1980), 358-383. MR 81c: 16027

[D1] N. I. Dubrovin, Noncommutative valuation rings, Trudy Moskov. Mat. Obshch. 45 (1982), 265-280; English transl. in Trans. Moscow Math. Soc. 45 (1984), 273-287. MR 85d:16002

[D2] N. I. Dubrovin, Noncommutative valuation rings in simple finite-dimensional algebra over a field, Mat. Sb. 123 (165) (1984), 496-509; English transl. in Math. USSR Sb. 51 (1985), 493-505. MR 85j: 16020

[E] O. Endler, Valuation Theory, Springer-Verlag, New York, 1972. MR 50:9847

[G1] J. Gräter, The Defektsatz for central simple algebras, Trans. Amer. Math. Soc. 330 (1992), 823-843. MR 92f: 16018

[G2] J. Gräter, Prime PI-rings in which finitely generated right ideals are principal, Forum Math. 4 (1992), 447-463. MR 93i:16026

[K] I. Kaplansky, Commutative Rings, The Univ. of Chicago Press 1970. MR 49:10674

[M1] P. J. Morandi, An approximation theorem for Dubrovin valuation rings, Math. Zeit. 207 (1991), 71-82. MR 92g:16021

[M2] P. J. Morandi, Maximal orders over valuation rings, J. Algebra 152 (1992), 313-341. MR 93k: 16028

[M3] P. J. Morandi, Noncommutative Prüfer rings satisfying a polynomial identity, J. Algebra 161 (1993), 324-341. MR 94k:16019 
[R] J. C. Robson, Rings in which finitely generated right ideals are principal, Proc. London Math. Soc. 17 (1967), 617-628. MR 36:200

[W] A. R. Wadsworth, Dubrovin valuation rings and Henselization, Math. Ann. 283 (1989), 301-328. MR 90f: 16009

Institute of Mathematics, Fudan University, Shanghai 200433, People's Republic of CHINA 